\title{
Maltrato físico y psicológico que genera el revoltijo en los estudiantes dentro de la universidad
}

\section{Physical and psychological abuse generated by the jumble in students within the university}

Johanna Enith Aguilar Reyes. ${ }^{1}$, Juan Efraín Sánchez Vimos. ${ }^{2}$, Fausto Francisco Navarrete Chávez. ${ }^{3}$ \& Verónica del Pilar Sánchez Alvear. ${ }^{4}$

Recibido: 27-05-2019 / Revisado: 15-06-2019 /Aceptado: 30-07-2019/ Publicado: 06-09-2019

\begin{abstract}
.
DOI: https://doi.org/10.33262/cienciadigital.v3i3.3.772

The jumble is a very old practice and nowadays it has become fashionable for many people who are not yet aware of the risk that constitutes. Here we can see the results, through the surveys applied to the students to be able to get an analysis of the topic. The jumble is a behavior that has accompanied the human being throughout its history, to start we can remember one of the most famous societies about it in history: The Romans; for almost all, it is known the sexual promiscuity that lived in ancient Rome, names like Tiberius, Caligula, Claudius, Julia and Messalina, are considered some of the great exponents of lust reigning in the Roman Empire. In this project that was made to the students of FIMA, we can examine respectively the jumble and abuse, with its possible causes, consequences, that these problems produce. The physical and psychological abuse occurs as a result of sexual practice at early ages without responsibility, with several people, known as jumble, not all students of the Polytechnic School of Chimborazo, Faculty of Sciences; they consider that this is so, due to the diversity of ideas and existing points of view, which will be evidenced with more specificity in the analysis that will be given in the next chapters of this research work.
\end{abstract}

Keywords: Jumble, abuse, lust, sexual, conduct, practice, current.

\footnotetext{
${ }^{1}$ Escuela Superior Politécnica del Chimborazo, Facultad de Ciencias. Riobamba, Ecuador, johannae.aguilar@espoch.edu.ec

${ }^{2}$ Escuela Superior Politécnica de Chimborazo, Facultad de Ciencias. Riobamba-Ecuador. jusanchez@espoch.edu.ec

${ }^{3}$ Escuela Superior Politécnica de Chimborazo, Facultad de Ciencias. Riobamba- Ecuador, fausto.navarrete@espoch.edu.ec

${ }^{4}$ Escuela Superior Politécnica del Chimborazo, Facultad de Ciencias. Riobamba- Ecuador. v_sanchez@espoch.edu.ec
} 


\section{Resumen}

El revoltijo es una práctica bien antigua y en la actualidad se ha convertido en moda para muchas personas que aún no tienen conciencia del riesgo que constituye.

Aquí podemos ver los resultados hechos, mediante las encuestas aplicadas a los estudiantes para poder sacar un análisis al tema tratado.

El revoltijo es una conducta que ha acompañado al ser humano durante toda su historia, para iniciar podemos recordar a una de las sociedades más famosas al respecto en la historia: Los Romanos; para casi de todos, es conocida la promiscuidad sexual en que se vivió en la antigua Roma, nombres como Tiberio, Calígula, Claudio, Julia y Mesalina, se consideran algunos de los grandes exponentes de la lujuria reinante en el imperio Romano.

En este proyecto que fue realizado a los estudiantes de FIMA, se puedo examinar respectivamente el revoltijo y maltrato, con sus posibles causas, consecuencias, que estos problemas producen.

El maltrato físico y psicológico se da como consecuencia de la práctica sexual a tempranas edades sin responsabilidad, con varias personas, conocida como revoltijo, no todos los estudiantes de la Escuela Superior Politécnica de Chimborazo, Facultad de Ciencias; consideran que esto sea así, por la diversidad de ideas y puntos de vista existentes, lo que se evidenciará con más especificidad en el análisis que se dará en los próximos capítulos de este trabajo de investigación.

Palabras claves: Revoltijo, maltrato, lujuria, conducta, sexual, practica, actualidad.

\section{Introducción.}

El siguiente proyecto acerca del revoltijo en que algunos casos que se dan en los estudiantes de FIMA, el mismo que surgió de la inquietud por conocer los factores que influían para que los jóvenes tomaran estas actitudes, sin considerar las consecuencias que se pueden presentar; Considerándolo de mucha importancia y de actualidad que debe ser meditada y sobe todo concientizada en los estudiantes. Siendo una de las posibles razones de los bajos rendimientos deserciones entre otros problemas sociales que se generan por esta forma desordena de actuar; Según el estudio realizado se presentó como una de las posibles causas y consecuencias para la deserción, por lo que se hace necesario realizar charlas que permitan mejorar la convivencia social entre los señores estudiantes. Con esta investigación nos daremos cuenta acerca del conocimiento sexual de las personas ya que en este país hablar del término REVOLTIJO o sencillamente de la sexualidad es un tema tabú el cual hoy en día es 
habitual encontrar en los diferentes medios de comunicación e internet gran cantidad de información sin ningún tipo de responsabilidad que permita aclarar dudas que se pueden presentar en la juventud siendo estos medios muy influyentes principalmente en los adolescentes que estén más propensos en estar inmiscuidos en situaciones de este tipo como: . Las enfermedades de transmisión sexual son muy comunes debido a las infidelidades en las parejas, y en los adolescentes simplemente por la curiosidad o el libertinaje que poseen. La carencia de valores es un factor influyente el ser promiscuo, porque es en el hogar donde se motivan los principios de una persona íntegra, pero es en el mismo hogar donde hace falta la presencia de educación sexual. Con esta investigación podremos darnos cuenta de cada detalle de por qué es importante tener una pareja estable y cuáles son las consecuencias de las infidelidades y del libertinaje.

\section{Metodología.}

Se va a describir el marco teórico sobre el tema en estudio el cual se refiere al revoltijo en los jóvenes universitarios, se investigó respecto al tema, en varias fuentes de informaciones tanto primarias como secundarias, las cuales se utilizaron de apoyo en esta investigación.

\section{El Revoltijo}

El revoltijo es una práctica bien antigua y en la actualidad se ha convertido en moda para muchas personas que aún no tienen conciencia del riesgo que constituye. Cambiar constantemente de pareja es una conducta que puede calificarse de suicida, generalmente estos encuentros esporádicos o de ocasión no implican lazos afectivos duraderos, y son muestras de uno de los comportamientos más aventurados para contraer las infecciones de transmisión sexual (ITS) y el VIH-SIDA.

La real academia española define la promiscuidad como la persona que mantiene relaciones sexuales con varias personas, así como de su comportamiento, modo de vida. A esto habría que añadirle que esa actitud no les supone ningún problema de tipo moral o psicológico, ya que es consecuente con sus actos y disfruta plenamente de sus relaciones.

Hoy día es difícil controlar las relaciones sexuales de nuestros jóvenes. Las jóvenes parejas mantienen estas relaciones sin necesidad de estar casados, y sin necesidad de cumplir la edad que antes era norma. La mayoría de estas jóvenes se sienten mal después de los encuentros sexuales, sintiéndose utilizadas, reflexionando en cómo pudieron haberse dejado convencer en entregar algo tan preciado a cambio de nada.

\section{Historia y Religión}

La promiscuidad es una conducta que ha acompañado al ser humano durante toda su historia, para iniciar podemos recordar a una de las sociedades más famosas al respecto en la historia: 
Los Romanos; para casi de todos, es conocida la promiscuidad sexual en que se vivió en la antigua Roma, nombres como Tiberio, Calígula, Claudio, Julia y Mesalina, se consideran algunos de los grandes exponentes de la lujuria reinante en el imperio Romano. Esa "libertad sexual" en que se vivía, no era sólo el privilegio de los gobernantes. La presencia de esclavos y esclavas en los hogares de los grandes señores permitía que se relacionaran sexualmente y también era algo bastante conocido por todo el mundo romano.

Lo que podríamos denominar "libertad sexual", estaba íntimamente relacionada con el amplio desarrollo de la prostitución.

Para la mayoría de las religiones, la promiscuidad es lo contrario a la castidad. En el ámbito social se considera lo contrario a la monogamia, aunque también existe el término bigamia, que especifica la actividad sentimental y por ende sexual con dos personas simultáneamente. Por ello podría asumirse que una persona promiscua mantiene relaciones sexuales con más de tres parejas, aunque en realidad no existe un número mínimo de compañeros sexuales para definir la promiscuidad. (wikipedia.org)

Promiscuidad es un término que puede variar entre culturas y que ha variado también en la historia. Para religiones como el cristianismo, el islamismo o el judaísmo, la promiscuidad es un acto escandaloso, ya que estas valoran el matrimonio y la monogamia. No todas las religiones comparten esta opinión: el budismo tiene una visión distinta al sexo e incluso a la prostitución. El islamismo en su momento permitía, de acuerdo al Corán, que los varones pudiesen tener varias esposas, aunque esto no se considera promiscuidad, porque el hombre debía mantenerlas a todas por igual.

\section{Causas del revoltijo}

Según el artículo de los factores que influyen en el revoltijo (tareas, 2010). Es importante conocer si factores como una mayor libertad sexual, concepto de sexualidad, diferencia entre madurez física y cognoscitiva y la tendencia a imitar los modelos adultos en los medios de comunicación influyen en la promiscuidad en la adolescencia para poder reducir los riesgos provocados por enfermedades. Los jóvenes prefieren ignorar las graves consecuencias que el sexo puede causarles, tales como enfermedades transmitidas, embarazos indeseados, o simplemente sentirse utilizadas. Aun así, lo que más les importa son las relaciones sexuales, que para ellos son experiencias que viven libremente, sin compromiso alguno. Esta forma de vivirlo puede acarrear inseguridad, sobre todo en la joven mujer que en su afán de sentirse amada se entrega más y más al juego del sexo, muchas veces sin antes llegar a la adultez. (Los riegos que genera el revoltijo, 2011)

Al entrar en la pubertad aumenta la preocupación por la sexualidad y el aspecto físico, esto puede verse influido por los medios de comunicación, así como el entorno del adolescente. 
Al inicio de la adolescencia es frecuente la masturbación y las fantasías sexuales que en ocasiones son con personas del mismo sexo, lo que genera sentimientos de culpa. La masturbación es más frecuente en los hombres que generalmente están motivados por la curiosidad.

Más tarde se dan los primeros encuentros físicos, aunque no necesariamente incluyen el coito, generalmente este contacto se da como una forma de exploración y aventura donde existe el intercambio de besos y caricias que más tarde darán pie al coito.

Actualmente son muchos los factores que influyen en la conducta sexual adolescente y que lo llevan a la práctica promiscua de su sexualidad; entre estos podemos encontrar la discrepancia entre la madurez física y la madurez cognoscitiva, esto es muy notorio en las jóvenes adolescentes en las cuales a muy temprana edad ocurre la menarquía y su cuerpo deja de ser el de una niña para convertirse en el de una mujer, aunque a nivel cognoscitivo sus pensamientos siguen siendo los de niña con la diferencia que ahora tendrá que jugar el juego que su cuerpo adulto "debe" seguir según cómo lo ve en su entorno.

A esto podemos sumar diversas condiciones sociales que hacen que la mayoría de los jóvenes no dejen de ser adolescentes o "hijos de familia" y permanezcan al "cuidado de sus padres" hasta por encima de los 25 o 30 años, algunas de estas condiciones son el alargamiento de los estudios y la dificultad para conseguir vivienda. Por otro lado, la percepción de invulnerabilidad que tienen los adolescentes de sí mismos los pone en un gran riesgo, ya que el sentimiento de inmortalidad favorece las conductas de riesgo tales como la promiscuidad sin protección ni responsabilidad.

La libertad sexual que ahora experimentan los adolescentes puede ser un factor que influye en el inicio precoz de la actividad sexual en los adolescentes ya que se ha comprobado que tanto la educación en un ambiente muy permisivo como la educación con normas muy rígidas o autoritarias conducen a un inicio en las relaciones sexuales de manera precoz. Por otro lado, se ha asociado el inicio precoz de relaciones sexuales en la adolescencia con una mayor incidencia de problemas ginecológicos y psicosociales en la vida adulta (actitud negativa hacia su propio cuerpo, conductas fuera de lo normal, etc.)

\section{Consecuencias del revoltijo}

Es preocupante que los adolescentes mantengan conductas sexuales de riesgo como lo es la promiscuidad ya que no se preocupan por las consecuencias que esto puede traerles a corto o largo plazo, algunas de estas consecuencias son los embarazos adolescentes o el contagio de enfermedades de trasmisión sexual. 
Las enfermedades de transmisión sexual (ETS) son un fiel reflejo de una sexualidad irresponsable; es decir, de la mantención de relaciones sexuales con distintas parejas, sin las prevenciones necesarias.

Para prevenir estas enfermedades es importante ser responsable de la propia sexualidad, considerando los siguientes factores: tener una pareja sexual estable, cuidar la salud genital, controlarse con un médico periódicamente y usar preservativos, sobre todo en el caso de relacionarse sexualmente con personas desconocidas.

Si alguien descubre que padece alguna de estas enfermedades, debe evitar tener relaciones sexuales y su pareja tendrá que ser analizada para detectar si está infectada.

\section{Las ETS más comunes son: Gonorrea}

Es una enfermedad venérea muy frecuente, fácil de detectar en el hombre, ya que se produce una secreción purulenta por la uretra con bastante dolor al orinar.

En cambio, en la mujer es mucho más difícil de descubrir, porque generalmente no da molestias, y si las da, no son muy específicas y pueden confundirse con otras infecciones genitales. Se produce una secreción purulenta por la vagina. Si no se trata, esta bacteria irá infectando y destruyendo los órganos genitales internos de la mujer, tales como el útero y las trompas de Falopio. Como consecuencia, a muchas mujeres les provoca esterilidad, o incapacidad de concebir un hijo.

\section{Sífilis}

Al igual que otras enfermedades venéreas, solamente se contagia por el contacto sexual con una persona infectada. Se puede descubrir fácilmente si luego de varios días de haber tenido una relación sexual aparece en los genitales una úlcera indolora de base dura, acompañada de inflamación en los ganglios de la ingle. Si ello ocurriera, se debe acudir rápidamente a un médico para un tratamiento que es ciento por ciento efectivo. De lo contrario, desaparecerá la úlcera y la infección se diseminará por todo el organismo, dañándolo de manera irreversible.

\section{EI VIH}

Es una de las enfermedades de transmisión sexual donde el 20\% de los casos se contrajo en la adolescencia. Hoy en día existe un incremento de riesgo provocado por tal práctica si no es realizada con responsabilidad y puede generar problemas en el futuro tales como un incremento en el riesgo de contraer infecciones de transmisión sexual y embarazos no deseados que pueden terminar en abortos inducidos, tal como lo señala (la Secretaria de 
ISSN: 2602-8085

www.cienciadigital.org

Vol. 3, N³.3, p. 77 - 94, septiembre, 2019

Salud; 2007) en el 2005 el $21 \%$ de los partos atendidos en instituciones públicas fueron en menores de 20 años y casi 7,289 ocurrieron en niñas de 10 a 14 años de edad por lo que se vuelve importante realizar el siguiente estudio.

La promiscuidad o tener una pareja sexual después de otra, elimina el auto respeto.

Las relaciones sexuales sin pensar en las consecuencias dejan heridas que al principio no las sienten, pero que más tarde sólo son reproches por no haber sabido esperar.

Las mujeres no somos objetos sexuales para ser explotadas para simplemente sentir placer. Si no pones en práctica el autocontrol, y por el contrario comienzas desde una temprana edad a experimentar el sexo, terminarás haciéndote adicta.

\section{Tipos de Promiscuidad:}

\section{Promiscuidad activa:}

Indica una condición sexual psicológica y comportamental de la persona que vive plenamente en la promiscuidad como tal, es decir, una sexualidad libre acorde al hedonismo, el sexo ocasional o casual con varias personas sin ningún tipo de ataduras de ninguna índole (afectiva o económica) y que también puede tener participación activa en orgías, frecuentar prostíbulos y fiestas de tipo sexuales.

\section{Promiscuidad pasiva}

Indica una condición sexual psicológica de cualquier persona cuyos actos no siempre son acordes a su sexualidad voluntaria, ya que puede estar condicionada o reprimida por factores culturales, de sociedad, religión, responsabilidad de cualquier tipo o por pudor. Generalmente son más responsables y controlados con su sexualidad permitiendo organizarse teniendo relaciones pasionales y afectivas. La promiscuidad pasiva se asocia muchas veces con la infidelidad, donde pueden presentarse en algunos casos bigamia o poligamia, y que también clandestina u ocasionalmente, cuando se le presenta la oportunidad, actúa como promiscuo activo. (Wikipedia, 2010).

\section{Marco Metodológico}

Esta parte de nuestra investigación detalla las hipótesis planteadas, la matriz de variables, el tipo de diseño y el tipo de estudio que se utilizó en la investigación, además de la muestra, la población y las técnicas utilizadas para analizar los datos estadísticos.

\section{Hipótesis}

a. Las principales causas de la promiscuidad entre los jóvenes estudiantes son:

- Libertinaje sexual 
- Inmadurez juvenil

- Influencia de los medios de comunicación

- Falso concepto de sexualidad

- Machismo

- Falta de valores

b. Las principales consecuencias de la promiscuidad son:

- Enfermedades de transmisión sexual

- Embarazos no deseados

- Abortos

- Impacto emocional negativo

c. El porcentaje de la promiscuidad en los jóvenes de la ESPOCH es muy alto.

- Las edades en que los jóvenes son más propensos a ser promiscuos, es entre los 15 y los 21 años de edad.

- Los más propensos a ser promiscuos son los hombres de 19

\section{Variables e Indicadores}

Las variables e indicadores están planteadas de acuerdo a los objetivos planteados en la investigación.

Definición conceptual Promiscuidad: La promiscuidad es una práctica bien antigua y en la actualidad se ha convertido en moda para muchas personas que aún no tienen conciencia del riesgo que constituye ya que se pueden adquirir enfermedades venéreas tales como: Gonorrea, Sífilis, VIH y más.

Libertinaje sexual: es la práctica superficial y sin límites del erotismo y conlleva a uno o más de los siguientes estados: aventuras rápidas, prostitución, disfunciones y desviaciones.

Machismo: El machismo, expresión derivada de la palabra "macho", se define en el DRAE como la "actitud de prepotencia de los varones respecto a las mujeres" El machismo engloba el conjunto de actitudes, conductas, prácticas sociales y creencias destinadas a promover estructuras tradicionales de familia y posición de la mujer. Algunos críticos consideran también machismo la discriminación contra otros grupos sociales, como en el caso de hombres homosexuales, como no "masculino. 
Feminismo: El feminismo es un conjunto heterogéneo de ideologías y de movimientos políticos, culturales y económicos que tienen como objetivo la igualdad de derechos entre varones y mujeres.

\section{Tipo de Diseño}

Esta investigación está realizada de acuerdo al diseño transversal no experimental ya que los datos se han recolectados en un solo momento y en un tiempo único, sin manipular deliberadamente las variables que fueron propuestas al inicio de la investigación. Además, su propósito será describir las variables y analizar su incidencia y su interrelación en un momento dado pues consiste en hacer énfasis en una sola medición de los datos obtenidos.

\section{Tipo de estudio}

La investigación sobre la promiscuidad en función de la profundidad en que se han establecido los objetivos es de tipo descriptivo y comparativo. Es comparativo porque se hará una comparación entre la información obtenida de las investigaciones ya realizadas acerca de la promiscuidad y la información con el análisis de datos de este estudio. Es de tipo descriptivo por que se pretende conocer las causas y consecuencias de la promiscuidad entre los estudiantes universitarios además de determinar el porcentaje de jóvenes que son promiscuos, establecer que género es más promiscuo e identificar las edades en las que los jóvenes son más propensos a la promiscuidad.

\section{Población y Muestra}

a. Tipo de muestra

En esta investigación se utilizó una muestra Probabilística de tipo racimo ya que va a ser realizada en la ESPOCH la cual pertenece a una comunidad, se dio por partes o etapas las cuales se desarrollaron y ejecutaron paulatinamente. Se seleccionó la muestra y se aplicó el instrumento al azar en las aulas de clase de la ESPOCH.

b. Tamaño de la muestra: Nuestra investigación será realizada en una población de 488 estudiantes de la ESPOCH, Facultad de Ciencias, Escuela de Física y Matemática Se utilizó una muestra de racimo de 215 estudiantes con un nivel de confianza del $95 \%$ y con un margen de error muestral del 0.5 .

\section{Técnica empleada.}

La investigación acerca de la promiscuidad en los estudiantes universitarios fue realizada a través de una encuesta con preguntas cerradas y precisas que se aplicó a la muestra seleccionada de 216 estudiantes de la ESPOCH / FIMA con el propósito de conocer la opinión que tienen los estudiantes de dicho tema además de cumplir con los objetivos planteados al iniciar el estudio. 


\section{Instrumentos Utilizados}

Se utilizaron los siguientes instrumentos:

- Encuestas impresas (véase anexo 1)

- Computador

- Calculadora

- Apuntes de clase

\section{Aspectos Administrativos}

\section{Recursos humanos, materiales, financieros}

Como recursos humanos estamos los integrantes del grupo, contribuimos para que el proyecto de investigación, con el cual al establecer ciertas hipótesis y gracias a la investigación, para la cual necesitamos de ciertos servicios: internet, copias, medios de trasporte (el cual usamos para trasladarnos a los lugares donde se realizaron las encuestas, lugares de encuentro para el desarrollo del proyecto, intercambio de ideas, criterios, discusión de resultados), el cual está de un gasto de alrededor de $20 \$$.

\section{Cronograma de actividades}

Figura 1. Diagrama De Gantt

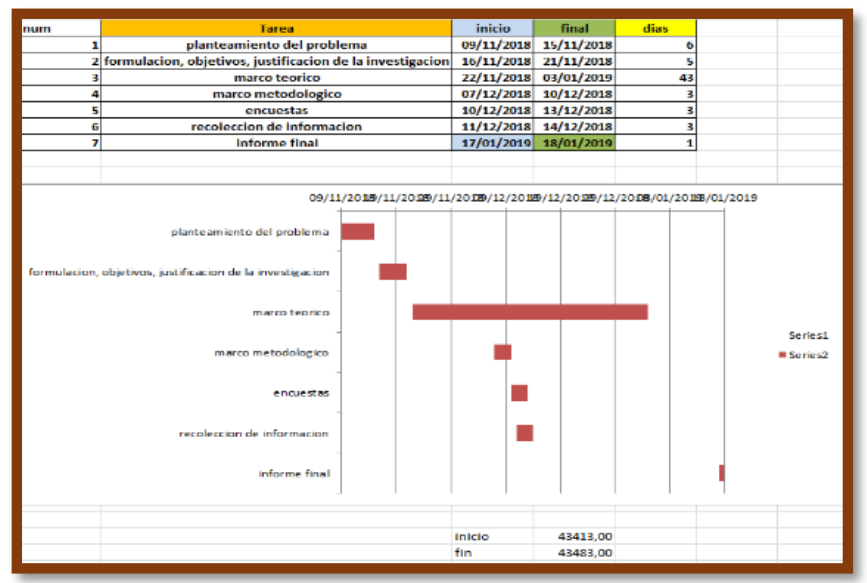

\section{Análisis}

En la presente sección se va a dar a conocer cuáles son los resultados obtenidos de las encuestas realizadas a los estudiantes de la Escuela Superior Politécnica de Chimborazo,

Facultad de Ciencias, Escuela de Física Matemática, con la finalidad de analizar los datos del tema tratado, con el propósito de saber si existe o no resultados sumamente altos respecto al problema planteado, por lo que se utilizara las técnicas más factibles para dar unos resultados 
más eficientes, para poder llevar a cabo este análisis se realizó una encuesta de 12 preguntas referente a la información requerida para llegar a una conclusión respecto al problema.

Tabla 1. Pregunta 1

\begin{tabular}{lcc}
\hline Etiquetas & $\begin{array}{l}\text { Cuenta de 1. ¿Tiene una vida sexual } \\
\text { activa? }\end{array}$ & $\%$ \\
\hline No & 61 & $28 \%$ \\
Hombre & 30 & \\
Mujer & 31 & $72 \%$ \\
Si & 155 & \\
Hombre & 86 & \\
Mujer & 69 & $100 \%$ \\
Total general & 216 & \\
\hline
\end{tabular}

Fuente: Elaboración propia

\section{Análisis de la pregunta 1. ¿Tiene una vida sexual activa?}

Analizando la información recopilada en base a las encuestas que hemos realizado, podemos decir que el $28 \%$ de los estudiantes que compren hombres y mujeres encuestadas no tiene una vida sexual activa y por otra parte existe el $72 \%$ de los estudiantes encuestados que, si la poseen, por lo que se puede concebir que más del $50 \%$ de los estudiantes entre hombres y mujeres tienen una vida sexual activa.

Tabla 2. Pregunta 2

\begin{tabular}{lll}
\hline Etiquetas de fila & Cuenta de 2. iA qué edad inicio su vida sexual? & $\mathbf{\%}$ \\
\hline 10 & 1 & $0,5 \%$ \\
11 & 2 & $0,9 \%$ \\
12 & 1 & $0,5 \%$ \\
13 & 5 & $2,3 \%$ \\
14 & 13 & $6,0 \%$ \\
15 & 23 & $10,6 \%$ \\
16 & 32 & $14,8 \%$ \\
17 & 42 & $19,4 \%$ \\
18 & 38 & $17,6 \%$ \\
19 & 14 & $6,5 \%$ \\
20 & 13 & $6,0 \%$ \\
21 & 1 & $0,5 \%$ \\
22 & 1 & $0,5 \%$ \\
Vacío & 30 & $13,9 \%$ \\
Total general & $\mathbf{2 1 6}$ & $100,0 \%$ \\
\hline
\end{tabular}

Fuente: Elaboración propia 


\section{Análisis de la pregunta 2. ¿A qué edad inicio su vida sexual?}

Según el resultado obtenido respecto a las encuestas realizadas podemos decir que existen 42 estudiantes que iniciaron su vida sexual a los 17 años con un porcentaje del 19,4\% del total, aquí podemos visualizar que la edad mínima que iniciaron los estudiantes su vida sexual es de 10 años y su edad máxima es de 22 años con un porcentaje de $0,5 \%$ respectivamente, por lo que podemos decir que inician su vida sexual desde los 10 años hacia delante.

Tabla 3. Pregunta 3

\begin{tabular}{lll}
\hline Etiquetas de fila & Cuenta de 3. ¿Con que frecuencia tiene relaciones sexuales? & \% \\
\hline A veces & 125 & $58 \%$ \\
Nunca & 40 & $19 \%$ \\
Siempre & 51 & $24 \%$ \\
Total general & $\mathbf{2 1 6}$ & $100 \%$ \\
\hline
\end{tabular}

Fuente: Elaboración propia

\section{Análisis de la pregunta 3. ¿Con que frecuencia tiene relaciones sexuales?}

En base a las encuestas realizadas podemos decir hay 125 estudiantes que tienen una vida sexual activa, y se puede decir que existe el 58\% de los estudiantes de la Escuela de Física y Matemática que tiene una vida sexual activa.

Tabla 4. Pregunta 4

\begin{tabular}{ll}
\hline $\begin{array}{l}\text { Cuenta de 4. ¿Ha sido discriminado o criticado por tener } \\
\text { dos o más parejas a la vez? }\end{array}$ \\
\hline 156 & $72 \%$ \\
60 & $28 \%$ \\
$\mathbf{2 1 6}$ & $100 \%$ \\
\hline
\end{tabular}

Fuente: Elaboración Propia

Análisis de la pregunta 4. ¿Ha sido discriminado o criticado por tener dos o más parejas a la vez?

En base a los datos recolectados podemos llegar a la conclusión que hay estudiantes que no han sido discriminados por tener dos o más parejas a la vez con un porcentaje del $72 \%$ y la cantidad de 156 estudiantes, pero no por él hay que descartar a los pocos estudiantes que han sido discriminados por tener dos o más parejas a la vez con un porcentaje del $28 \%$ y con la cantidad de 60 estudiantes. 
Tabla 5. Pregunta 5

\begin{tabular}{llll}
\hline $\begin{array}{l}\text { Etiquetas } \\
\text { fila }\end{array}$ & de & $\begin{array}{l}\text { Cuenta de 5. ¿Considera usted a la promiscuidad como un } \\
\text { maltrato físico o psicológico? }\end{array}$ & \% \\
\hline No & 150 & $69 \%$ \\
$\mathrm{Si}$ & 66 & $31 \%$ \\
Total general & $\mathbf{2 1 6}$ & $100 \%$ \\
\hline
\end{tabular}

Fuente: Elaboración propia

Análisis de la pregunta 5 ¿Considera usted a la promiscuidad como un maltrato físico o sicológico?

Interpretando cada uno de los resultados obtenidos en las encuestas se puede manifestar o decir que el $31 \%$ de los estudiantes con una cantidad de 150 en la respuesta no, consideran que la promiscuidad no es un maltrato físico o psicológico y solo una pequeña parte dice que, si es un maltrato psicológico con una cantidad de 66en la respuesta si, que es el $31 \%$ del total.

Tabla 6. Pregunta 6

6. ¿Cuáles de los métodos anticonceptivos conoce o acostumbra a usar?

\begin{tabular}{lllll}
\hline & $\begin{array}{c}\text { Cuenta de } \\
\text { Preservativo }\end{array}$ & $\begin{array}{c}\text { Cuenta de } \\
\text { Pastillas de } \\
\text { emergencia }\end{array}$ & $\begin{array}{c}\text { Cuenta de } \\
\text { Pastillas } \\
\text { anticonceptivas }\end{array}$ & $\begin{array}{c}\text { Cuenta de } \\
\text { Inyección } \\
\text { Anticonceptiva }\end{array}$ \\
n & 163 & 59 & 62 & 40 \\
$\%$ & $75 \%$ & $27 \%$ & $29 \%$ & $19 \%$ \\
\hline
\end{tabular}

Fuente: Elaboración propia.

\section{Análisis de la pregunta 6 ¿Cuáles de los métodos anticonceptivos conoce o acostumbra} a usar?

En este análisis podemos ver que existen 163 estudiantes que utilizan o conocen el preservativo con un $75 \%$ del total, también se toma en cuenta las patillas de emergencia, las patillas anticonceptivas y la inyección Anticonceptiva con una cantidad de 59,62y 40 estudiantes respectivamente con los porcentajes de $27 \%, 29 \%$ y el $19 \%$ del total.

Aquí no podemos dar cuenta que la mayoría de los estudiantes conocen o han usado el preservativo, teniendo en cuenta que es muy fácil de adquirir.

Tabla 7. Pregunta 7

\begin{tabular}{lll}
\hline Etiquetas de fila & $\begin{array}{l}\text { Cuenta de 7. iHa tenido relaciones } \\
\text { sexuales forzadas? }\end{array}$ & $\%$ \\
\hline No & 200 & $93 \%$ \\
$\mathrm{Si}$ & 16 & $7 \%$ \\
Total general & $\mathbf{2 1 6}$ & $100 \%$ \\
\hline
\end{tabular}

Fuente: Elaboración Propia 


\section{Análisis de la pregunta 7 ¿Ha tenido relaciones sexuales forzadas?}

Mediante el análisis hecha por la información recopilada en las encuestas se puede decir que la mayoría de los estudiantes encuestados no han tenido relaciones sexuales forzadas y esto comprende los 200 estudiantes con el 93\%, pero también hay que tomar en cuenta a la pequeña cantidad que, si han tenido relaciones sexuales forzadas que comprende a 16 estudiantes con un $7 \%$, que hay que tener muy en cuenta.

Tabla 8. Pregunta 8

\begin{tabular}{lll}
\hline Etiquetas de fila & $\begin{array}{l}\text { Cuenta de 8. iHa sido agredida física } \\
\text { psicológicamente? }\end{array}$ \\
\hline A veces & 24 & $\mathbf{\%}$ \\
Nunca & 189 & $11 \%$ \\
Siempre & 3 & $88 \%$ \\
Total general & $\mathbf{2 1 6}$ & $1 \%$ \\
\hline
\end{tabular}

Fuente: Elaboración propia

\section{Análisis de la pregunta 8 ¿Ha sido agredida física o psicológicamente?}

De acuerdo con el análisis realizado tenemos que decir que hay una mínima máxima cantidad de estudiantes de la Escuela de Física y Matemática que no son maltratados ni física ni sicológicamente que conforman el $88 \%$ del total con una cantidad de 189 estudiantes al contrario que hay 27 estudiantes que si son maltratados y 3 de ellos son maltratados siempre y la otra parte a veces.

Tabla 9. Pregunta 9

\begin{tabular}{lll}
\hline Etiquetas de fila & \multicolumn{2}{l}{$\begin{array}{l}\text { Cuenta de 9. Si su respuesta anterior fue } \\
\text { afirmativa ¿quién le agredió? }\end{array}$} \\
\hline Amigos & 6 & $\mathbf{\%}$ \\
Mamá & 2 & $3 \%$ \\
Otros ¿Quién? & 6 & $1 \%$ \\
Papá & 3 & $3 \%$ \\
Pareja & 8 & $1 \%$ \\
Vacío & 191 & $4 \%$ \\
Total general & $\mathbf{2 1 6}$ & $88 \%$ \\
\hline
\end{tabular}

Fuente: Elaboración Propia 


\section{Análisis de la pregunta 9 su respuesta anterior fue afirmativa ¿quién le agredió?}

Mediante los datos obtenidos de las encuestas podemos decir que existe a la mayoría de los estudiantes no son maltratados ya que esta pregunta se complementa con la que vimos anteriormente , pero aquí también aumentan las personas que si son maltratadas y no quieren avisar quien lo hizo, por lo que tenemos más personas que no sabemos quién las maltrata, por el otro lado tenemos a los amigos con el $3 \%$ en la totalidad de 6 , mamá con el $1 \%$ con la totalidad de 2,papá con el 1\% con la totalidad de 3 ,pareja con el $4 \%$ con la totalidad de 8 , y otras personas ajenas a las mencionadas anteriormente, con la totalidad de 6 , por lo que no se puede descartar que hay maltrato en los estudiantes de FIMA

Tabla 10. Pregunta 10

\begin{tabular}{cccccc}
\hline & \multicolumn{5}{c}{$\mathbf{1 0 .}$ ¿Qué enfermedades de trasmisión sexual conoce? } \\
\cline { 2 - 6 } & $\begin{array}{c}\text { Cuenta de } \\
\text { Clamidia }\end{array}$ & $\begin{array}{c}\text { Cuenta de } \\
\text { Herpes genital }\end{array}$ & $\begin{array}{c}\text { Cuenta de } \\
\text { Sífilis }\end{array}$ & $\begin{array}{c}\text { Cuenta de } \\
\text { VIH/SIDA }\end{array}$ & $\begin{array}{c}\text { Cuenta de } \\
\text { Gonorrea }\end{array}$ \\
$\mathbf{N}$ & 56 & 134 & 114 & 199 & 134 \\
$\%$ & $26 \%$ & $62 \%$ & $53 \%$ & $92 \%$ & $62 \%$ \\
\hline
\end{tabular}

Fuente: Elaboración propia

\section{Análisis de la pregunta 10. ¿Qué enfermedades de trasmisión sexual conoce?}

Mediante la pregunta analizada se puede decir que la mayoría de los estudiantes de FIMA conocen sobre la enfermedad del VIH/SIDA ya que tenemos con un $92 \%$ de su totalidad con una cantidad de estudiantes de 199, en comparación de las otras enfermedades de transmisión sexual que poseen un porcentaje de $26 \%$ de la Clamidia, el 62\% del herpes genital, el 53\% de sífilis, el $62 \%$ de gonorrea cada uno con la totalidad de estudiantes de 56, 134, 114,134 respectivamente.

Tabla 11. Pregunta 11

\begin{tabular}{lll}
\hline Etiquetas de fila & $\begin{array}{l}\text { Cuenta de 11. iPor qué tienes } \\
\text { relaciones sexuales? }\end{array}$ & $\mathbf{\%}$ \\
\hline Compromiso sentimental & 45 & $21 \%$ \\
Obligación & 7 & $3 \%$ \\
Placer & 134 & $62 \%$ \\
Vacío & 30 & $14 \%$ \\
Total general & $\mathbf{2 1 6}$ & $\mathbf{1 0 0 \%}$ \\
\hline
\end{tabular}

Fuente: Elaboración propia. 


\section{Análisis de la pregunta 11 ¿Por qué tienes relaciones sexuales?}

En base a lo analizado se pues decir que la mayoría de las personas tiene o a tenido relaciones sexuales por placer con una cantidad de 134 estudiantes y el porcentaje considerable al $62 \%$, con esto se puede decir que es más del 50\% que lo hace por esta razón “placer”, pero no hay que olvidar sobre la otra parte del porcentaje que compre el compromiso sentimental, por obligación que es el porcentaje del $21 \%$ y el $3 \%$ respectivamente y la última parte que pertenece a las personas que no han tenido relaciones sexuales o simplemente se limitan a decir cuál es la razón.

Tabla 12. Pregunta 12

\begin{tabular}{lll}
\hline Etiquetas de fila & $\begin{array}{c}\text { Cuenta de 12. En base a las preguntas } \\
\text { hechas iUsted se considera una persona } \\
\text { promiscua? }\end{array}$ & $\%$ \\
\hline No & 164 & $76 \%$ \\
Hombre & 84 & \\
Mujer & 80 & $24 \%$ \\
Si & 52 & \\
Hombre & 32 & \\
Mujer & 20 & $100 \%$ \\
\hline
\end{tabular}

Fuente: Elaboración Propia

\section{Análisis de la pregunta 12. En base a las preguntas hechas ¿Usted se considera una persona promiscua?}

En base a los resultados obtenidos en las encuestas podemos decir que la mayoría de los estudiantes no se consideran promiscuos y esto está conformado por el $76 \%$ con la cantidad de estudiantes de 164, por la otra parte tenemos que el $24 \%$ de los estudiantes si se considera promiscuos entre hombres y mujeres forman una totalidad de 52 estudiantes.

\section{Conclusiones.}

- Se puede observar que el revoltijo se presenta más en los hombres que en las mujeres de la carrera, cosas que se evidencia en los estudios realizados anteriormente.

- En el desarrollo de esta investigación se llegó a evidenciar que existe tanto maltrato físico como maltrato psicológico por parte de la respectiva pareja.

- Al realizar este tipo de investigación se pudo evidenciar que este fenómeno alcanza a grandes grupos especialmente al sector estudiantil, ya que por unas u otras razones ellos tienden a tener práctica incorrecta con su vida sexual. 
- Se debe escoger la manera correcta de comportarse dentro de este hábito, debido a que al no estar prevenidos muy bien sobre los riesgos se puede tener consecuencias muy negativas.

- Prevenir antes de lamentar es algo que debemos tener en cuenta, porque aún tenemos la vida por delante y hay muchas cosas que se debe hacer y cumplir.

\section{Referencias bibliográficas.}

Barradas, E. O. (16 de diciembre de 2008). Estudios en sociología. Recuperado el 19 de febrero de 2013, de www.actoressociales.com.mx

Carranza, M. (05 de septiembre de 2012). Libertinaje y Promiscuidad. Recuperado el 20 de febrero de 2013, de www.dea.gob.gt

García, D. (2010). Actualidad lésbica. Pensamiento Femenino.

Pineda, C. (29 de agosto de 2012). Universidad Manuela Beltrán. Recuperado el 15 de febrero de 2013, de www.umb,ed.com

tareas, B. (2010). Planeación de investigación sobre conductas sexuales. 05.

http://www.todamujeresbella.com/4543/el-peligro-de-la-promiscuidad/

http://www.todamujeresbella.com/2651/mujeres-promiscuas/ (2011, 07). Los riesgos que genera la promiscuidad. BuenasTareas.com. Recuperado 07, 2011, de http://www.buenastareas.com/ensayos/Los Riesgos que genera la promiscuidad/2571021.html

Somos jóvenes digitales. (abril de 2007). Recuperado el 15 de febrero de 2013, de http://www.somosjovenes.cu/index/semana60/campareja.htm

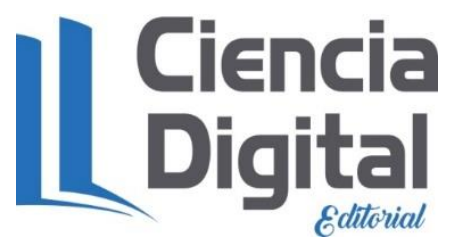




\section{PARA CITAR EL ARTÍCULO INDEXADO.}

Aguilar Reyes, J., Sánchez Vimos, J., Navarrete Chávez, F., \& Sánchez Alvear, V. del P. (2019). Maltrato físico y psicológico que genera el revoltijo en los estudiantes dentro de la universidad. Ciencia Digital, 3(3.3), 77-94. https://doi.org/10.33262/cienciadigital.v3i3.3.772

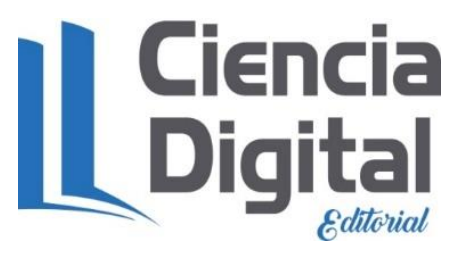

El artículo que se publica es de exclusiva responsabilidad de los autores y no necesariamente reflejan el pensamiento de la Revista Ciencia Digital.

El artículo queda en propiedad de la revista y, por tanto, su publicación parcial y/o total en otro medio tiene que ser autorizado por el director de la Revista Ciencia Digital.
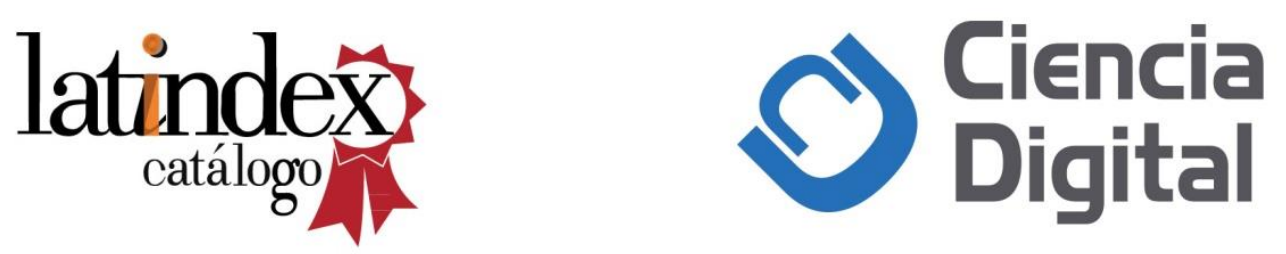